de las Iglesias; b) información del pueblo en los paises en desarrollo ofreciéndoles las condiciones para el establecimiento de un diálogo real. Esta es, en verdad, una tarea muy importante para el trabajo de communicación social de las Iglesias.

\title{
Die Zukunft der katholischen Sonntagspresse im Licht einer Leser-Umfrage
}

\author{
von Ferdinand Oertel
}

\begin{abstract}
Niemand bestreitet, daß die Presse trotz des Vordringens der visuellen und akustischen Medien noch eine sichere Zukunft hat. Wenn sich auch die Strukturen und Gestaltungsformen der bisherigen Zeitungen und Zeitschriften immer mehr ändern und aus wirtschaftlichen Gründen Konzentrationen und Kooperationen erfolgen, so bleibt doch die Tatsache eines ständig wachsenden Lese-Bedürfnisses des heutigen Menschen bestehen. Nicht von ungefähr erleben gegenwärtig die Publikumszeitschriften, die vorwiegend der Unterhaltung dienen, eine unerwartete Auflagensteigerung.

Nicht ohne Grund haben die deutschen Bischöfe 1968 die Gründung einer neuen katholischen Wochenzeitschrift für wichtig erachtet. Es ist zwar nicht so, als ob es bislang keine katholische Presse in Deutschland gegeben habe oder gibt, aber die wenigen, ausgesprochen katholischen Tages- und Wochenzeitungen politischer Ausprägung spielten im Nachkriegskonzert der deutschen Presse leider nie eine große Rolle, und der übrigen kirchlichen Presse warf (und wirft) man vielfach vor, sie sei nicht wirksam genug.
\end{abstract}

\section{Kirchliche Presse in der Krise}

Unter der sogenannten kirchlichen Presse versteht man die Bistumszcitungen und die Sonntagsblätter, die Verbandszeitschriften und die Ordens- sowie Missionsblätter. Diese Presse hat immerhin auch heute noch eine Gesamtauflage von mehr als $12 \mathrm{Mil}-$ lionen Exemplaren, ist in ihrer Verbreitung aber in der Tat ausschließlich auf den innerkirchlichen Raum begrenzt. Die Vorwürfe an sie, provinziell und altmodisch zu sein, nur von braven Kirchgängern bezogen und kaum gelesen zu werden, kann man heute nicht mehr aufrechterhalten. Der weitaus größte Teil dieser Zeitschriften müht sich um eine zeitgemäße, wenn auch nicht avantgardistische Aufmachung und um eine offene Darstellung und Behandlung aller religiösen Gegenwartsproblematik. Dennoch sinkt die Abonnentenzahl dieser Blätter ständig, und im Gegensatz zur allgemeinen Presse sehen sie sich ernsthaft vor die Frage gestellt, ob sie noch eine Zukunft haben.

Die Beantwortung dieser Frage wird entscheidend davon abhängen, ob der einzelne Mensch und die Gesellschaft in Zukunft noch eine so enge kirchliche Bindung eingehen wie bisher. Viele Anzeichen deuten darauf hin, daß dies nicht der Fall ist.

Dr. Ferdinand Oertel ist Chefredakteur des katholischen Sonntagsblattes „Die christliche Familie", das in Essen erscheint. 
Die Sorge um die Zukunft stellt sich für die verschiedenen Gruppen innerhalb der kirchlichen Presse jedoch unterschiedlich dar. Für die von den Bischöfen oder Bistümern herausgegebenen offiziellen bzw. offiziösen Kirchenzeitungen dürfte entscheidend werden, welchen Freiheitsraum sie für den offenen innerkirchlichen Dialog erhalten. Die Zukunft der Verbandsorgane hängt eng mit dem Fortleben dieser Verbände zusammen, während für die Ordens- und Missionspresse entscheidend wird, ob sie in Zukunft neue tragfähige und zeitgemäße Formen ihrer Aufgabenstellung finden kann. Bei der katholischen Sonntagspresse scheint die Situation etwas anders zu liegen, da sie keinen kirchlichen Herausgeber hat. Von der Zeit ihrer Gründung an nahm sie im katholisch-kirchlichen Pressebereich immer eine Sonderstellung ein. Ihre Tradition reicht größtenteils sogar weiter zurück als die der Verbandsorgane und der Bistumsoder Kirchenzeitungen. Beachtenswert ist dabei, daß diese Blätter alle der privaten Initiative katholischer Laien entsprangen und bis heute private verlegerische Unternehmen geblieben sind. So sind sie einerseits nicht gebunden an kirchliche Richtlinien, haben andererseits aber auch keine direkte kirchliche Unterstützung. $\mathrm{Da}$ es bei diesen Überlegungen besonders um die Zukunftsaussichten dieser Sonntagsblätter gehen soll, muß ihr geschichtlicher Standort kurz umrissen werden.

\section{Die Situation der Sonntagsblätter}

Mitte des 19. Jahrhunderts meinten die Gründer dieser Sonntagsblätter, der liberalen "Gartenlauben"-Presse, aber auch der zum Teil nicht sehr glaubens- und kirchenfreundlichen Tages- und Unterhaltungspresse, andere Zeitschriften gegenüberstellen zu müssen, die auf dem festen Standpunkt des katholischen Glaubens ruhten und sich an das breite Kirchenvolk wandten. Zu den Wochenzeitschriften, die, wenngleich nicht alle in so früher Zeit gegründet, dennoch aus jenem Impuls Leben gewannen, zählen "Das Bayerische Sonntagsblatt" (München), "Die christliche Familie" (Essen), das "Liborius-Blatt" (Hamm), der "Altöttinger Liebfrauenbote" und "Nach der Schicht" (Wiebelskirchen/Saar).

Alle diese Zeitschriften waren typische katholische Familienblätter, brachten vorwiegend unterhaltende Beiträge mit viel Moral, aber auch belehrende und kämpferische Zeitbetrachtungen. Sie wollten das Kirchenvolk im Glauben stärken und standen also von Anfang an ganz im Dienste der Kirche. Damit erfüllten sie im Kulturkampf, aber auch im Kaiserreich vor dem Ersten Weltkrieg und selbst noch in der Weimarer Zeit eine wichtige Aufgabe des katholischen Presse-Apostolates. Insofern unterschieden sie sich dann auch kaum von den regionalen Kirchenzeitungen, die vor und nach dem Ersten Weltkrieg überall entstanden. Wie diese, mußten auch sie im Dritten Reich eingestellt werden.

Nach dem Zweiten Weltkrieg standen die wiedererscheinenden privaten Sonntagsblätter neben bischöflich herausgegebenen Bistumsblättern und richteten sich praktisch an dieselbe Leserschicht, wenn sie inhaltlich auch in den Grundakzenten etwas verschieden waren. Während die Bistumsblätter sich vorwiegend den kirchlichen Ereignissen im eigenen Bistum und in der Weltkirche widmeten (und dabei informationsmäßig auch die Lücke zu füllen suchten, die durch den Fortfall einer ausgeprägten katholischen Tagespresse entstanden war), dienten die Sonntagsblätter mehr der Unterhaltung und der allgemeinen katholischen Familienbildung.

In ihrer Zielsetzung, zur christlichen Lebensgestaltung beizutragen, stimmen die Sonntagsblätter auch heute mit der übrigen kirchlichen Presse überein. In weiten Kreisen der Leserschichten gelten sie immer noch als „Kirchenblätter". Auf dem Anzeigensektor werden sie genauso wie die Bistumsblätter zur konfessionellen Presse 
gezählt, und ihre Auflagenentwicklung verläuft in den letzten Jahren ebenso langsam nach unten oder stagniert. Für die Sonntagsblätter entsteht also die gleiche Frage wie für die kirchlich gebundene Presse: ob sie überhaupt noch eine Zukunft haben. Die Frage wird vielfach negativ beantwortet, vor allem mit der Behauptung, die Leserschaft dieser Blätter sei alt und sterbe langsam aus, während neue und jüngere Leser für sie nur schwer zu gewinnen seien, zumal ganz allgemein das Interesse an religiös gebundenen Lebensformen nachlasse. Voraussetzung für eine echte Beantwortung der Frage nach den Zukunftschancen dieser Zeitschriften ist jedoch eine genaue Kenntnis ihrer gegenwärtigen Leserstruktur und Lesermentalität.

Aus diesem Grund hat „Die christliche Familie“ (=CF) zwei Untersuchungen durchführen lassen: eine psychologische Untersuchung der Leserschaft durch ein Düsseldorfer Marketinginstitut im Oktober 1968 und eine eigene Leser-Befragung bei $6000 \mathrm{Be}-$ ziehern im Januar 1969. Im folgenden sollen daraus einige Ergebnisse bekanntgegeben werden, die für die weiteren UUberlegungen über die Zukunft der Sonntagsblätter aufschlußreich sind.

\section{Ergebnisse der Untersuchungen}

Die Druckauflage der CF betrug im IV. Quartal 1968 rund 196000 Exemplare. Die Bezieher setzen sich zu 65,5 Prozent aus Frauen und zu 34,5 Prozent aus Männern zusammen (Bundesdurchschnitt: 54 Prozent Frauen, 46 Prozent Männer). Rund 70 Prozent der Bezieher sind verheiratet, 16 Prozent verwitwet und nur 13 Prozent ledig. Dieses Bild ergibt eine typische "Familien"-Leserschaft.

Entgegen einer weitverbreiteten Meinung ist diese Leserschaft nicht überaltert. Zwar sind nur 6,4 Prozent der Bezieher unter 25, aber 17,6 Prozent liegen im Alter zwischen 25 und 35 Jahren und 31,9 Prozent zwischen 35 und 50 Jahren. Fast die Hälfte aller Bezieher zählt demnach zu den mittleren Generationen zwischen 25 und 50 Jahren (Bundesdurchschnitt ca. 46 Prozent). Da die Zahl der Kinder bis zu 15 Jahren in unmittelbarer Beziehung zum Generationsalter der Eltern steht, zeichnet sich auch von dieser Seite her das Schwergewicht einer „Familien“-Leserschaft mit Kindern bis zum Ende der Schulpflichtzeit ab.

Fast die Hälfte aller Bezieher wohnt in kleinen Orten bis zu 10000 Einwohnern, davon wieder 50 Prozent in Orten unter 3000 Einwohnern (Bundesdurchschnitt: 22 Prozent). Wenn damit auch die Zahl der Bezieher in den kleinen Orten höher liegt als im Bundesdurchschnitt, so entspricht die Zahl derjenigen, die in Kleinstädten ab 10000 und in Großstädten über 100000 Einwohnern leben, wiederum dem Bundesdurchschnitt. Auch einkommensmäßig liegen die Bezieher der CF in etwa mit dem Durchschnitt der Bundesbürger gleich.

Im Gegensatz zur allgemeinen Bevölkerungsstruktur überwiegt bei der Leserschaft der CF etwas die Zahl der Angestellten und Beamten gegenüber den Arbeitern: 34 Prozent der Bezieher sind Angestellte und Beamte, 22 Prozent Arbeiter. In ihrer sozialen Zusammensetzung wird man die Leserschaft als mittelschichtbetont ansehen müssen. Insofern dürfte sie sich nicht vom Bundesdurchschnitt unterscheiden, ist also keineswegs eine "aussterbende" Leserschaft, was Alter, soziale Situation und Bildung betrifft.

Ein Unterscheidungsmerkmal gegenüber dem Bundesdurchschnitt besteht jedoch in der religiösen Bindung: 92 Prozent der Leser sind katholisch. Gegenüber den anderen katholisch-kirchlichen Organen dürfte es ins Gewicht fallen, daß immerhin acht Pro- 
zent der Bezieher evangelisch sind. In ihrer Reichweite greift die CF also uiber den katholischen Bereich hinaus. Leider geht aus beiden Untersuchungen nicht hervor, wie viele der katholischen Leser zu den Kirchgängern zählen und wie viele vielleicht nur Randkatholiken sind. Ubereinstimmend sagen beide Analysen jedoch aus, daß nur jeder fünfte Bezieher auf kirchliche Empfehlung an das Blatt gekommen ist. Ein Drittel der Bezieher wurde durch Türwerbung gewonnen, ein weiteres Fünftel übernahm die Anregung zum Abonnement vom Elternhaus.

Die Tatsache, daß insgesamt die Hälfte aller Bezieher durch Kirche und Elternhaus an die Zeitschrift gekommen sind, besagt aber, daß bei dieser Gruppe eine starke Autoritätsbindung vorliegt. Was die Zeitschrift veröffentlicht, besitzt für sie einen hohen Wahrheitscharakter. Bei der psychologischen Untersuchung zeigte sich so auch eine starke Hemmung unter den Befragten zur offenen Kritik. Positiv formulierte Aussagen über das Blatt erhielten große Zustimmung, negative fanden kaum Zustimmung. Auf Grund der engen Verbindung zu Kirche und Elternhaus, die von mehr als der Hälfte der Bezieher ( $z$. T. unbewußt) hergestellt wird, steht die Zeitschrift vielfach außerhalb rationaler Kritik.

Dieses autoritätsbegründete „Vertrauen" wird bestätigt durch die Dauer der Abonnementsverhältnisse. 43 Prozent beziehen die Zeitschrift mehr als zehn Jahre, 41 Prozent zwischen zwei und zehn Jahren. Nur 9,2 Prozent sind im ersten Jahr Bezieher. Die Lese-Intensität liegt ebenfalls sehr hoch. 81 Prozent sagten aus, sie nähmen die Zeitschrift drei- und mehrmal zur Hand, und jeder zweite Bezieher bewahrt die Zeitschrift auf.

Auch durch ihr Verhältnis zu anderen Massenmedien sind die Leser dieser katholischen Familienzeitschrift besonders gekennzeichnet. 95 Prozent der Bezieher besitzen ein Radio, 90 Prozent ein Fernsehgerät. 79 Prozent lesen zusätzlich eine Tageszeitung. Damit sind die Hauptquellen von Information und Unterhaltung für die Mehrheit

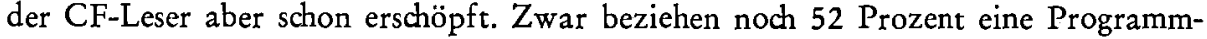
zeitschrift (eine Zahl, die in direkter Beziehung zu den vorhandenen Geräten steht), aber nur 27 Prozent lesen noch eine Illustrierte, nur 20 Prozent eine andere Frauenzeitschrift und nur fünf Prozent eine Boulevardzeitung. Uberraschend dürfte es sein, daß insgesamt auch nur 43 Prozent angaben, neben der CF noch eine andere kirchliche Zeitschrift $\mathrm{zu}$ beziehen. Das mag ein Hinweis darauf sein, daß die andere Gruppe der Leserschaft keine so starke Bindung an die Kirche hat wie die gleichzeitigen Kirchenzeitungsleser.

Die Zustimmung zur christlichen Grundhaltung der CF, die bereits aus dem geringen Prozentsatz der Leser anderer Publikumszeitschriften hervorgeht, wird durch mehrere andere Aussagen bestätigt. In der psychologischen Untersuchung sagten 93 Prozent aus, die Zeitschrift habe "eine gute moralische Haltung". 93 Prozent hoben hervor, $\mathrm{da} ß$ sie "nicht so marktschreierisch und aufdringlich wie andere Zeitschriften" sei, und 88 Prozent betonten, sie sei "nicht so kompliziert und hochgeschraubt wie andere". Die Leser-Umfrage ergab, daß nur 9,8 Prozent „mehr über Filmstars und Königshäuser" lesen möchten, während die anderen gerade lobten, daß die CF "nicht so sehr auf Sensationen" aus sei.

Im Mittelpunkt des allgemeinen Leseinteresses steht trotz der Anerkennung der christlichen Grundhaltung aber nicht die religiöse Thematik, sondern stehen eindeutig Unterhaltung und Erziehungs-Hilfen. Beide Untersuchungen ergaben, daß Romane, Erzählungen und Kurzweil an erster Stelle gelesen und gewünscht werden. Es folgen Erziehungsbeiträge, aktuelle Berichte und Familien-Artikel. Erst an fünfter Stelle kommen religiöse Beiträge. Auf der Wunschliste für eine Erweiterung der Zeitschrift 
stehen an erster Stelle "Praktische Dinge" (77 Prozent), gefolgt von Ehe und Familie (65 Prozent) sowie aktuelle Berichte (60 Prozent). Auf religiöse Beiträge legen 42 Prozent Wert, während im übrigen nur zwölf Prozent "mehr Bilder“" wünschen.

Diese Ergebnisse zeigen, daß die Bezieher der CF ihre Zeitschrift als ein „Blatt zum Lesen" mit Schwerpunkten bei Unterhaltung und Familienbildung werten und auch so gestaltet sehen möchten.

\section{Auswertungen und Ausblick}

Die beiden Untersuchungen über die gegenwärtige Leserstruktur und die Erwartungen an die Zeitschrift kommen im wesentlichen zu folgenden Ergebnissen:

1. Die Leserschaft entspricht in ihrer Struktur dem Durchschnitt der Bundesbürger. Sie ist weder überaltert noch gehört sie sozial oder bildungsmäßig einer aussterbenden Schicht an.

2. Die Leserschaft schätzt an der Zeitschrift die seriöse Grundhaltung in Inhalt, Stil und Gestaltung.

3. Etwa die Hälfte der Leser hat eine starke traditionelle Bindung an die Zeitschrift, die von den Faktoren Kirche und Elternhaus ausgeht. Wahrscheinlich ist es dieselbe Gruppe, die auch eine engere Bindung an die Kirche hat.

4. Die andere Hälfte der Leserschaft steht offensichtlich der Kirche ferner, wenngleich sie die christliche Basis bejaht.

Es ist nicht einfach, auf Grund dieser Ergebnisse Schlüsse für die Zukunft zu ziehen. Im Grunde ist die Frage gestellt, ob das Sonntagsblatt mehr der traditionsbewußten Leserschaft zugewendet bleiben soll, wobei der Grundcharakter einer "kirchlichen" Wochenzeitschrift beibehalten werden müßte, oder ob sie sich mehr jenem Leserkreis öffnet, für den das christliche Element nicht im Vordergrund steht, sondern der rein sachbezogene Inhalt. Aus der psychologischen Untersuchung scheint sich jedoch jene Richtung ablesen zu lassen, in der eine größere Zukunft liegt. Bei dieser Untersuchung wurde nämlich auch ein Vergleich $z$ wischen der "Christlichen Familie" und einer „idealen Zeitschrift" dieser Art gezogen. Danach wird die CF im Durchschnitt nicht gerade als ausgesprochen traditionsbewußt oder gar unmodern eingestuft, liegt für viele Leser aber doch noch weiter in der konservativen Richtung.

Den wesentlichen Unterschied kennzeichnet jedoch die Frage der "religiösen Bindung": Die Einstufung für eine "ideale Zeitschrift" tendiert bei den Befragten nur ganz schwach in Richtung auf "christlich-religiös“, wogegen die CF jetzt noch als eindeutig so bezeichnet wird. Der Wunsch nach weniger religiöser Bindung ist dabei bei den Lesern, die erst kürzere Zeit Abonnenten sind, und bei den jüngeren weitaus größer als bei den älteren und vieljährigen Abonnenten. Die Gruppe der jüngeren Bezieher wünscht gleichzeitig stärkere Aktualität und mehr Dinge der praktischen Lebensgestaltung.

Will man ein Fazit ziehen, dann scheint in dieser Richtung die besondere Chance der Sonntagsblätter zu liegen. Sie müssen sich gleichsam ganz zur Welt hin öffnen oder konkreter gesagt: dem Alltag des Menschen unserer modernen Gesellschaft. Vor allem im Hinblick darauf, daß diese Gesellschaft vor immer größere Probleme der Freizeitbewältigung gestellt wird, kommt den Medien der Lebensgestaltung eine neue Bedeutung zu. Wenn es stimmt, daß das Freizeitproblem den Menschen dann auch auf eine neue Fragestellung nach dem Sinn des Lebens führt, hat die Sonntagspresse eine Aufgabe wahrzunehmen, die durch andere kirchliche Organe für die in Frage kom- 
mende Leserschicht nicht wahrgenommen werden kann. In gewissem Sinne muß die Sonntagspresse sich also vielleicht ganz vom Image einer kirchlichen oder religiösen Presse freimachen, um in der „weltlichen" Gesellschaft für die Kirche und das Glaubensleben wirken zu können.

Ob sie diesem Anspruch in der jetzigen Form und Struktur überhaupt entsprechen kann, ist eine andere Frage. Vielleicht wird bei einer anderen Preisgestaltung, bei einer verschärften Anzeigensituation oder bei erschwerten Bedingungen für die Bezieherwerbung (die jetzt ausschließlich durch Türwerbung geschehen muß), eine stärkere Kooperation oder Konzentration nicht mehr lange ausbleiben können. Die $\mathrm{Zu}$ sammenlegung verschiedener deutscher Sonntagsblätter und Halbmonats- oder Monatsmagazine zu einem großen Familienwochenblatt wäre meines Erachtens durchaus denkbar und sowohl für die redaktionelle als auch für die verlegerische Leistung fruchtbar. Eine Zukunftschance besteht jedenfalls für diese Sparte von der Aufgabe her ebenso wie vom Lesermarkt.

\section{S U M MARY}

There is still a special kind of Catholic Sunday press in Western Germany. Initiated as a reaction to liberal and socalled inferior weeklies in the 19th century, it is asked in this article by Dr. Ferdinand Oertel, editor in chief of "Die christliche Familie“ (The Christian Family) circulation: 199,300-if there will be still a chance for this type of magazine in the future. Dr. Oertel reports on two inquiries started by his publication. From these he concludes that the Catholic Sunday press has a duty to their readers in the future which can't be fulfilled by other Church related papers. Perhaps the image of this Sunday press has to be changed to a more secular direction in order to serve more efficiently the Church and faith.

\section{RE S U M E N}

La prensa dominical católica de la República Federal de Alemania conoce un tipo de revistas de caracter particular que tiene sus raices en la reacción católica contra la liberal y la así denominada "mala" prensa semanal del siglo XIX. Dirigida todavía hoy principalmente a distraer y formar a las familias católicas, estas publicaciones dominicales justifican el interrogativo de sus posibilidades en el futuro que el Doctor Fernando Oertel, redactor jefe de "La Familia Cristiana" (tirada : 199.300 ejemplares), Essen, nos presenta en esta colaboración. En ella nos presenta el resultado de dos encuestas que su revista realizó entre sus lectores. Llega a la conclusión que la prensa dominical tiene también en el futuro una misión, la cual, dadas las estructuras de los lectores, no puede ser conseguida por otros órganos periodísticos eclesiásticos. Es posible que tenga que cambiar su imagen, en el sentido de secularizarse más, a fin de poder ejercer un influjo más efectivo sobre la Iglesia y en la vida de la fe.

\section{CDU- nahe Lizenzzeitungen (IV): „Kölnische Rundschau“}

\section{von Heinz-Dietrich Fischer}

Den Ruf Kölns als Zeitungsstadt hielten vor 1933 die traditionsreiche liberale „Kölnische Zeitung" und das rheinische Zentrumsorgan, die „Kölnische Volkszeitung“, aufrecht. Die Traditionen der katholischen Presse, die bis in die erste Hälfte des 19. Jahrhunderts zurückreichen ${ }^{1}$, ließen Köln nach dem deutschen Zusammen- 